\title{
F5N : Nanopore Sequence Analysis Toolkit for Android Smartphones
}

\author{
Hiruna Samarakoon*, Sanoj Punchihewa*, Anjana Senanayake*, Roshan Ragel* and Hasindu Gamaarachchi ${ }^{\dagger}$ \\ * Department of Computer Engineering, University of Peradeniya, Sri Lanka \\ $\dagger$ School of Computer Science and Engineering, UNSW Sydney, Australia and \\ Kinghorn Centre for Clinical Genomics, Garvan Institute of Medical Research, Australia
}

\begin{abstract}
F5N is the first ever Android application for nanopore sequence analysis on a mobile phone, comprised of popular tools for read alignment (Minimap2), sequence data manipulation (Samtools) and methylation calling (F5C/Nanopolish). On NA12878 nanopore data, F5N can perform a complete methylation calling pipeline on a mobile phone in $\sim 15$ minutes for a batch of 4000 nanopore reads $(\sim 34$ megabases). $F 5 N$ is not only a toolkit but also a framework for integrating existing $\mathrm{C} / \mathrm{C}++$ based command line tools to run on Android. F5N will enable performing nanopore sequence analysis on-site when used with an ultra-portable nanopore sequencer (eg: MinION or the anticipated smidgION), consequently reducing the cost for special computers and high-speed Internet.
\end{abstract}

Availability and implementation : F5N Android application is available on Google Play store at https://play.google.com/ store/apps/details?id=com.mobilegenomics.genopo\&hl=en and the source code is available on Github at https://github.com/SanojPunchihewa/f5n.

Contact : hirunas@eng.pdn.ac.lk

\section{INTRODUCTION}

Ultra-portable single-molecule real-time sequencers such as MinION introduced by Oxford Nanopore Technologies (ONT) measure the ionic current when a DNA strand passes through a biological nanopore [1]. The sequencer periodically outputs a group of reads in the form of raw current signals (packed into a fast 5 file) which are subsequently base-called (to a fastq file) on a laptop or an ultra-portable ONT's MinIT. Therefore, sequencing and base-calling processes have become portable but sequence analyses such as sequence alignment and genome polishing are still not. Currently, the sequence analysis is either performed using a cloud service (that involves uploading data over high-bandwidth network) or using dedicated high-end server computers, both of which are not synonymous with ultra-portable sequencing. We present the first ever mobile nanopore DNA sequence analysis toolkit $F 5 N$, which compacts popular DNA analysis tools to an Android mobile phone application to make sequence analysis fully portable (Fig. 1A).

The ultra-portable MinION sequencer has proven to be beneficial for in-the-field sequencing in a variety of environments, such as Ebola surveillance in West Africa [2], microbial communities inspection in the Arctic [3] and DNA sequencing on the International Space Station [4]. The scarcity of portable and offline analysis solutions restricts the true potential of these sequencers. For instance, during Ebola surveillance, scientists encountered unexpected Internet connectivity issues where $3 \mathrm{G}$ signals dropped to $2 \mathrm{G}$, massively increasing the data upload time. Mobile applications like $F 5 N$ would be beneficial in such instances. In addition, mobile applications like $F 5 N$ have the potential to increase the value of the ONT's SmidgION, the upcoming smartphone pluggable sequencer.

$F 5 \mathrm{~N}$ can execute an analysis process (known hitherto as the pipeline) on a nanopore dataset copied or downloaded by the user on to the phone storage. Out of the available tools Minimap2, Samtools and F5C, the user can select to run an individual tool, a combination of tools or the whole methylation detection pipeline (Fig. 1B). The intuitive graphical user interface in the application allows the user to configure the most common parameters for tools (Fig. 1C). A terminal environment is also provided in the application for an advanced user to provide command-line arguments. When the user starts the execution, the output is written to the phone storage as specified by the user and the log output is displayed on the application in real-time (Fig. 1D).

To get familiar with $F 5 N$, an example demonstration is provided inside the application which automatically downloads and executes a complete methylation calling pipeline on a small test dataset. $F 5 N$ not only supports smaller genomes (eg: bacteria and virus) but also large genomes such as the human genome through an index partitioning approach as demonstrated in [5]. Refer Supplementary Sections II,III or the Help in the Android application on how to use F5N.

\section{Methodology}

F5N Android Application (GUI and the framework) was developed using Java programming language. Popular longread aligner Minimap2 [6], the sequence data manipulator Samtools [7] and the methylation caller F5C [8] (optimised version of the popular tool Nanopolish [9]) were re-configured and cross-compiled to produce shared libraries (.so files) to run on Android over the ARM processor architecture. The interface between the Android Java application and the native code (compiled shared libraries) was written using the Java Native Interface (JNI). This interface invokes the native functions in the compiled libraries and captures the log output using Unix Pipes. The captured output is displayed on F5N GUI. Android Software Development Kit (SDK) and the Android Native Development Kit (NDK) were used as development tools.

One can re-configure different other bioinformatics tools written in $\mathrm{C} / \mathrm{C}++$ by following the detailed methodology in Supplementary Section VI and integrate them to $F 5 N$ by following the guide in Supplementary Section VII. Thus, $f 5 c$ is not just a toolkit, but also a framework for integrating existing or future $\mathrm{C} / \mathrm{C}++$ based command line bioinformatics tools. The challenges imposed by the restrictions in Android Operating system along with the methods to overcome them are discussed in Supplementary Section VIII.

\section{BENCHMARK RESULTS}

We benchmarked $F 5 N$ using two publicly available NA12878 nanopore MinION datasets (flowcell IDs FAB42804 and FAF05869) [10]. A complete methylation calling pipeline was executed: read alignment to the full human reference genome (GRCh38) using Minimap2 (8 index partitions), sorting and indexing of the alignments using Samtools followed by methylation calling using F5C (Supplementary Section I). Benchmarking was performed on four different phones (device 


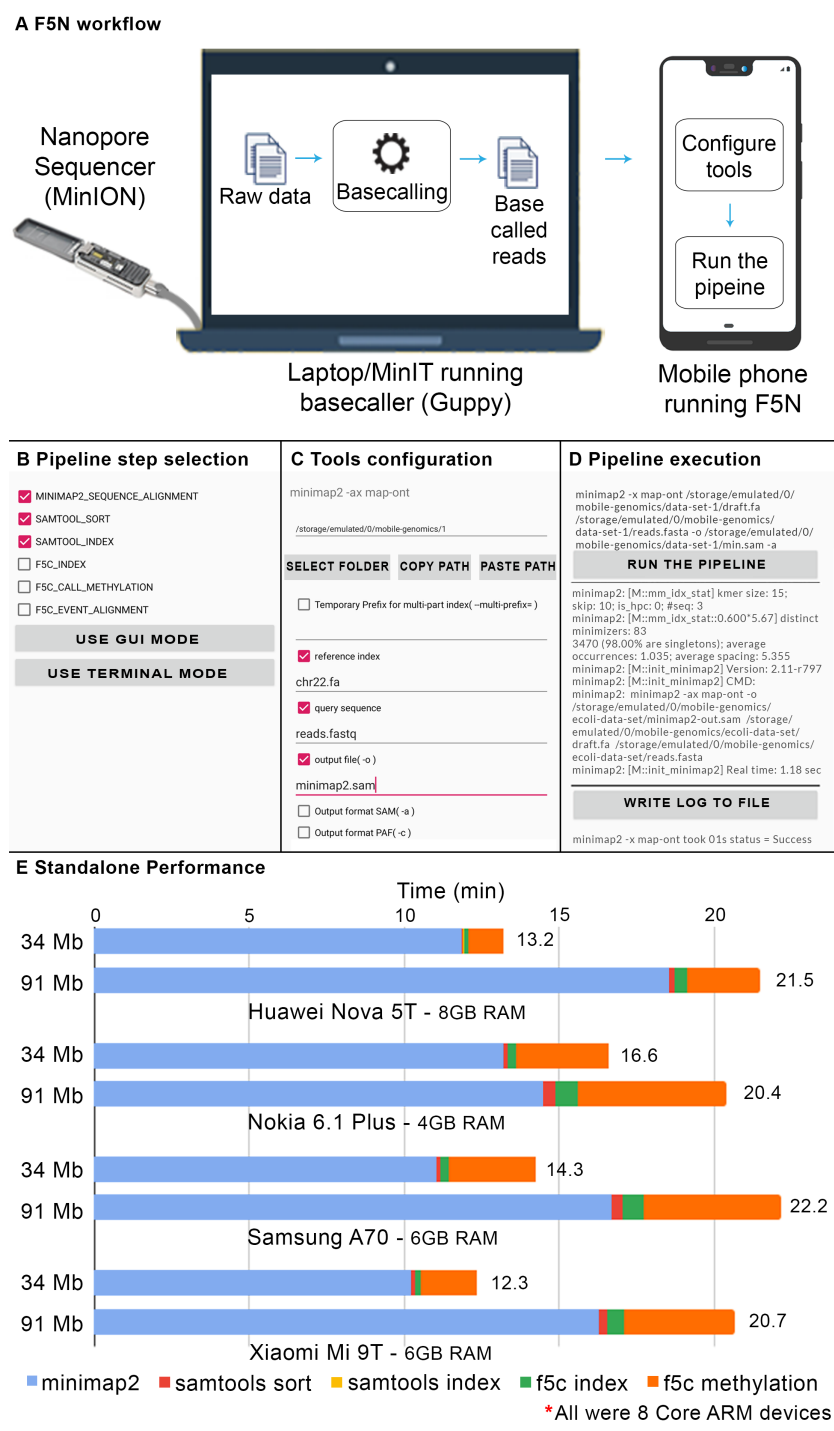

Fig. 1. (A) Real-time analysis work setup. MinION connected basecaller and basecalled data transferred to a mobile device. (B,C,D) Screenshots of $F 5 N$. (E) Performance evaluation on four smartphones for two datasets (91 Mbases and 34 Mbases)

specification in Supplementary Table S1). For full experiment details refer Supplementary Section III.

FAB42804 MinION dataset being comparatively smaller (only 16688 reads, 91.15 Mbases), F5N took 21.18 minutes in average to complete the whole methylation calling pipeline on it (Fig. 1E and Supplementary Table S2). A percentage of $77.8 \%$ of the execution time was consumed by Minimap2 read alignment and $18.0 \%$ percentage of the time was consumed by $F 5 C$ methylation calling. The highest and the second highest peak RAM were recorded for F5C methylation calling (3.4 GB) and Minimap2 reads alignment $(2.2 \mathrm{~GB})$ respectively. By adjusting the memory governing parameters of each tool, peak RAM can be reduced to support a variety of low end devices (Supplementary Section IV).

FAF05869 MinION dataset (451,359 reads, 3.89 Gbases) was used to create batches of 4000 reads $(\sim 34.49$ Mbases per batch on average) to mimic the batch processing behaviour of the base-caller. Batches of reads were assigned on to all four mobile phones and were processed in parallel. A total time of 14.09 minutes was recorded in average for a single batch to complete the pipeline (Fig. 1E and Supplementary Table S4). A percentage of $89.8 \%$ of the execution time was for Minimap 2 read alignment. The highest average peak RAM of $3.0 \mathrm{~GB}$ was recorded for Minimap2 read alignment (Supplementary Table S5). Given a sequencing run on the MinION takes 48 hours, a single mobile phone would have been adequate to perform analysis on-the-fly for this particular dataset (with a power source to keep the phone charged) - one batch could be processed in 14.09 minutes while the sequencer would produce a batch only after every 25.52 minutes in average (see Supplementary Section V). Our future work will focus on harnessing the computing power of multiple mobile phones in parallel to keep-up with sequencing runs that produce larger data volumes.

\section{CONCLUSION}

F5N demonstrates the true potential of portable genomics by executing a complete nanopore methylation calling pipeline locally on an Android mobile phone. A batch of 4000 nanopore reads ( $\sim 34$ megabases) could be processed in $\sim 15$ minutes where read alignment to the human genome, sorting and methylation calling consumed $\sim 12,<1$ and $\sim 2$ minutes, respectively. $F 5 N$ can also be used by the community as a framework for integrating other tools and pipelines for nanopore data analysis. As future work, we will extend $F 5 N$ to seamlessly connect multiple mobile phones to the base-calling device (laptop or ONT MinIT) through Wi-Fi, to process data even faster and on-the-fly during a sequencing run.

\section{ACKNOWLEDGMENTS}

Many thanks to Chandima Samarasinghe, Harshana Weligampola, Nirodha Suchinthana, James Ferguson, Rahal Medawatte, and Yasiru Ranasinghe for providing valuable feedback after testing $F 5 N$.

\section{REFERENCES}

[1] Hengyun Lu, Francesca Giordano, and Zemin Ning. "Oxford Nanopore MinION sequencing and genome assembly". In: Genomics, proteomics \& bioinformatics 14.5 (2016), pp. 265-279.

[2] Joshua Quick et al. "Real-time, portable genome sequencing for Ebola surveillance". In: Nature 530.7589 (2016), pp. 228-232.

[3] Jacqueline Goordial et al. "In situ field sequencing and life detection in remote (79 26' N) Canadian high arctic permafrost ice wedge microbial communities". In: Frontiers in microbiology 8 (2017), p. 2594.

[4] Sarah L Castro-Wallace et al. "Nanopore DNA sequencing and genome assembly on the International Space Station". In: Scientific reports 7.1 (2017), pp. 1-12.

[5] Hasindu Gamaarachchi, Sri Parameswaran, and Martin A Smith. "Featherweight long read alignment using partitioned reference indexes". In: Scientific reports 9.1 (2019), p. 4318.

[6] Heng Li. "Minimap2: pairwise alignment for nucleotide sequences". In: Bioinformatics 34.18 (2018), pp. 30943100 .

[7] Heng Li et al. "The Sequence Alignment/Map format and SAMtools". In: Bioinformatics 25.16 (June 2009), pp. 2078-2079.

[8] Hasindu Gamaarachchi et al. "GPU Accelerated Adaptive Banded Event Alignment for Rapid Comparative Nanopore Signal Analysis". In: bioRxiv (2019), p. 756122 .

[9] Jared T Simpson et al. "Detecting DNA cytosine methylation using nanopore sequencing". In: Nature methods 14.4 (2017), p. 407.

[10] Miten Jain et al. "Nanopore sequencing and assembly of a human genome with ultra-long reads". In: Nature biotechnology 36.4 (2018), p. 338. 


\title{
Supplementary Materials - F5N : Nanopore DNA Data Analysis Toolkit for Android Smartphones
}

\author{
LIST OF TABLES
}

S1

S2

S3

S4

S5

1 F5N project directory structure, only the important folders are shown. The directory cpp contains CMakeLists.txt and interface_X.h header files. The .so files are stored according their ISA inside jniLibs directory. The Java Class PipelineStep is inside directory core. . . . . . . . . . . . . . . . . . . . . . . . .

\section{Contents}

I Call methylation pipeline

II F5N Guide

III Detailed F5N examples and results

IV Adjusting memory governing parameters of the tools

V Caclulating base-calling rate

VI Reconstructing sequence analysis tools for ARM/Android

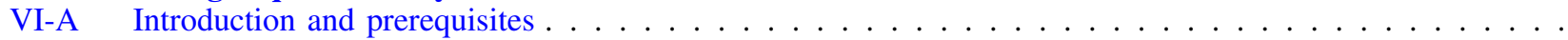

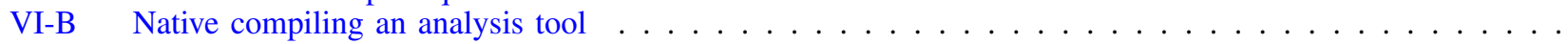

VI-C Determining third party libraries used by an analysis tool $\ldots \ldots \ldots \ldots$

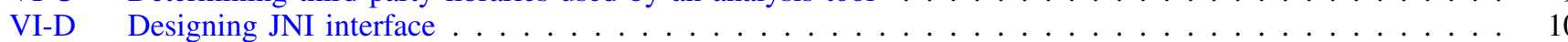

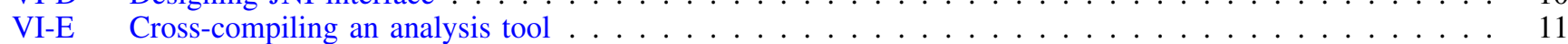

VI-F Dynamic object construction in Java side . . . . . . . . . . . . . . . . . 11

VII Integrating a new analysis tool to F5N

VIII Advanced details 
bioRxiv preprint doi: https://doi.org/10.1101/2020.03.22.002030; this version posted June 9, 2020. The copyright holder for this preprint (which was not certified by peer review) is the author/funder, who has granted bioRxiv a license to display the preprint in perpetuity. It is made available under aCC-BY-NC 4.0 International license.

I. Call methylation pipeline

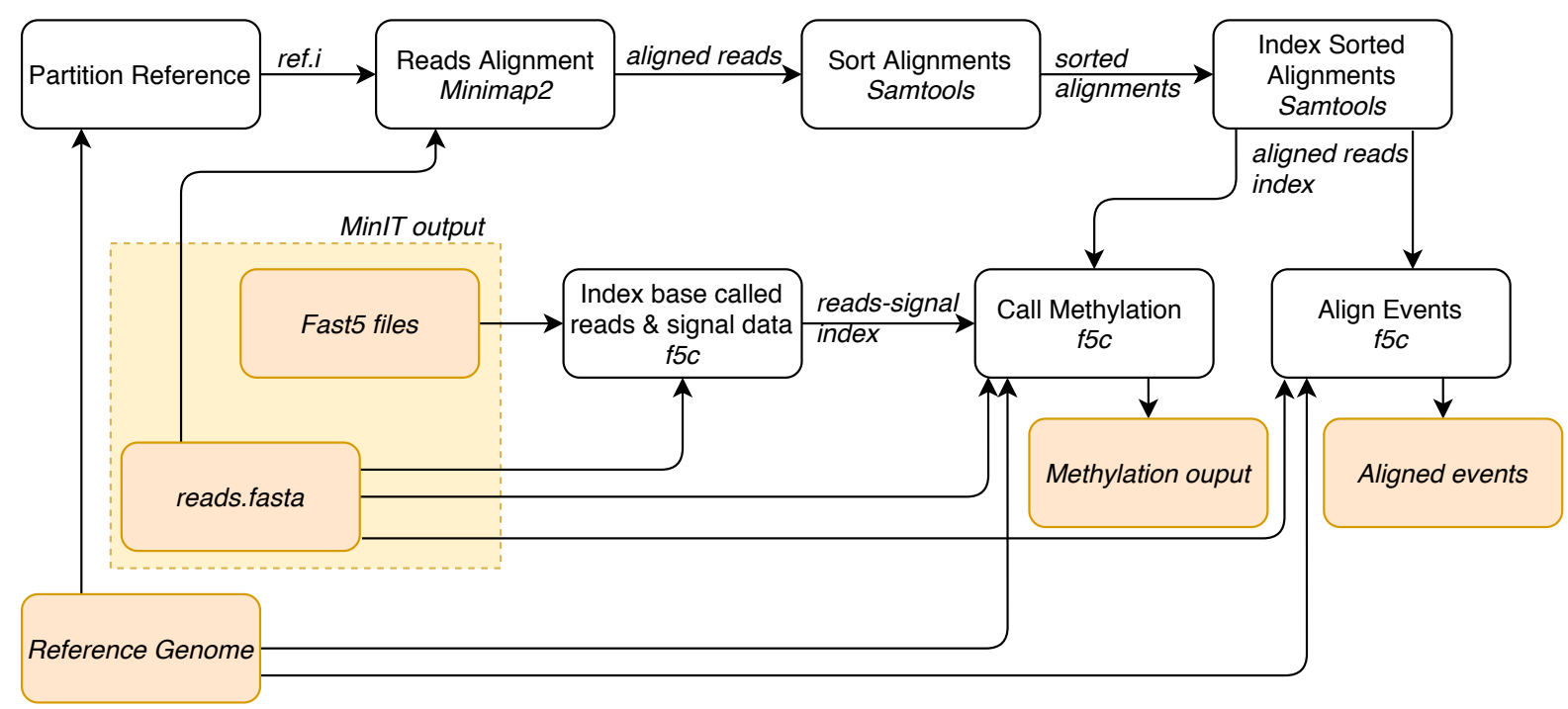

Fig. S1. Methylation Calling (and Event Alignment) Pipelines comprise of three analysis tools - Minimap2, Samtools and F5C. Partition reference step is only required for larger genomes that do not fit in RAM.

F5N supports two complete DNA analysis pipelines - methylation calling and event alignment. Both pipelines are very similar except for the last steps. In this paper, we explain only the methylation calling pipeline. The pipeline has six steps (Fig. S1). The pipeline's inputs are a reference genome, ONT raw signal data (.fast5 files) and the corresponding base called reads (reads.fastq(a) file). The first step of the pipeline is to align base called reads to the reference genome. The widely used reads alignment tool is Minimap2 [6] [11]. However, Minimap2's memory usage grows with the size of the reference genome. Since a mobile phone has limited memory, Minimap2 crashes for larger references. A novel algorithm was introduced to overcome the memory constraint using partitioned index [5]. We integrate this technique into our pipeline along with Minimap2. Please note that the reference partitioning should be done on a computer and the partitioned reference can be stored on a mobile phone (Supplementary Section III). Secondly, the aligned reads are sorted. Then these sorted results are indexed. Both sorting and indexing tasks use Samtools [7]. The final phase of the pipeline is polishing. Nanopolish [12] is the widely used polishing tool for nanopore data. We adopt a re-engineered version of Nanopolish called F5C [8], which is both memory and time efficient. F5C first indexes base called reads and raw signal data. Subsequently, $F 5 C$ can either perform methylation calling or event alignment. In Event alignment pipeline, event alignment step is performed instead of methylation calling. 


\section{F5N GUIDE}

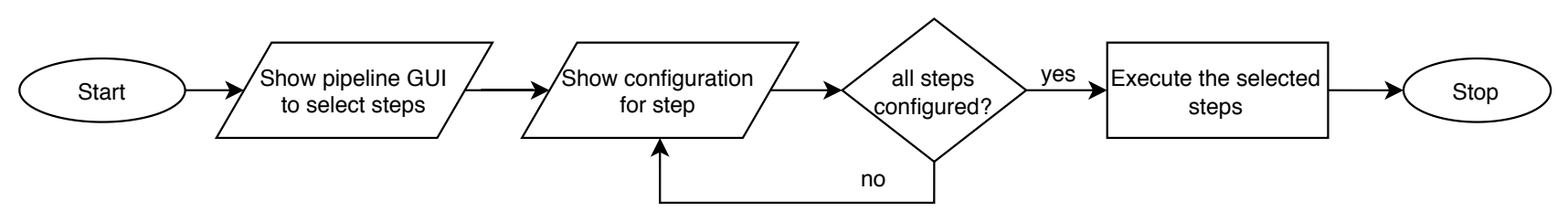

Fig. S2. F5N stand-alone mode work flow

$F 5 N$ has four major functionalities which are listed below.

1) Stand-alone mode for configuration and execution of a custom pipeline on the mobile device.

2) Mobile-cluster mode for real time analysis using a cluster of mobile devices, which is currently under development and will not be discussed in this paper.

3) Download data-sets using URLs and extract compressed files.

4) An example demonstration of downloading and extracting a nanopore data-set of E. Coli Bacteria, followed by executing a complete methylation calling pipeline on the data-set.

F5N includes a help section for new users to get started. It has a summary of the above four major functionalities (Fig. S3 B). Once $F 5 N$ mobile application is launched for the first time, the user is prompted to grant permission to read and write from the internal storage of the mobile device. In most of the devices, once this permission is set, it is adequate to read from and write to the external storage (SD card) as well. However, in certain devices the user is expected to set this permission explicitly, which can be done by navigating to [help $\rightarrow$ set SD card permission] section (Fig. S3 C).

F5N's start page has listed down the above four functionalities (Fig S3 A). A user navigating to stand-alone mode will land on a page to select custom pipeline steps from where he can choose Minimap2, Samtools, F5C or a desired combination of those tools (Fig. S3 F). Once the steps are selected, the user can choose either GUI mode or (Fig. S3 G and H) to configure parameters for each tool. Fig. S2 shows the procedure to use stand-alone mode. It is recommended to use the GUI mode as the final commands are always compiled into a set of strings and later shown in the terminal mode before proceeding to the execution. In GUI mode file path arguments get auto completed once the user set the correct path to the data set directory. F5N provides an elegant directory navigator for this purpose and both GUI mode and terminal mode have it. If the user chose terminal mode at the beginning, he skips GUI mode and lands on the terminal mode. From the terminal mode the user can proceed to the pipeline execution page (Fig. S3 I). Once the pipeline execution is started a timer will be displayed. After the execution of the pipeline the user can write results to a $\log$ file (named f5n.log) which is located inside storage/mobile-genomics directory. In the rare event of a crash of $F 5 N$, the user can run the previous pipeline using LOAD PREVIOUS CONFIGURATION command. If the app crashes during an execution, the user can identify the error occurred by referring tmp.log which is located inside storage/mobile-genomics folder. For more information regarding log files please refer the help section on home page in the application.

Functionalities to download a data-set form a URL and extract a compressed data-set are available on a same page (Fig. S3 D). To download a data-set, the user has to set the specific data-set URL path and the location on the storage to where the data-set should be downloaded. Decompressing a file is as easy as setting the file path of the compressed file and pressing the EXTRACT button. The decompressed file will have the same location as the compressed file's. Since a data-set usually consists of many numbers of considerably small fast 5 files, it will take much time to transfer them to a device storage unless the files are compressed. Hence, $F 5 N$ is provided with a file extraction functionality to decompress the files as necessary. In mobile-cluster mode compressed files will get transferred over WiFi.

The example demonstration is a setup with only three steps to help users get familiar with $F 5 N$. The steps involve the basic procedure to execute a pipeline. They are 1, download a data set 2, extract the data set and 3, execute the pipeline (Fig. S3 E). 
bioRxiv preprint doi: https://doi.org/10.1101/2020.03.22.002030; this version posted June 9, 2020. The copyright holder for this preprint (which was not certified by peer review) is the author/funder, who has granted bioRxiv a license to display the preprint in perpetuity. It is made available under aCC-BY-NC 4.0 International license.

A Mode Selection

STANDALONE

CONNECT TO MINIT

DOWNLOAD / EXTRACT DATASET

App Version 0.2.2

D Download and Extract

You can use this mode to download a data set (zip file) and extract it to a desired location

You can download a sample data set by pressing DOWNLOAD SAMPLE ECOLI DATSET

Once you have downloaded a zip file, select that file and press EXTRACT to unzip it

\begin{tabular}{|c|c|c|}
\hline \multicolumn{3}{|c|}{ Url of the data set } \\
\hline \multicolumn{3}{|c|}{ /storage/emulated/0/mobile-genomics } \\
\hline \multicolumn{3}{|c|}{ SELECT FOLDER } \\
\hline \multicolumn{3}{|c|}{ DOWNLOAD DATA } \\
\hline \multicolumn{3}{|c|}{ DOWNLOAD SAMPLE ECOLI DATASET } \\
\hline \multicolumn{3}{|c|}{$\begin{array}{l}\text { /storage/emulated/0/mobile-genomics/ } \\
\text { data-set-1.zip }\end{array}$} \\
\hline \multicolumn{3}{|c|}{ SELECT FILE } \\
\hline \multicolumn{3}{|c|}{ EXTRACT } \\
\hline \multicolumn{3}{|c|}{$\begin{array}{l}\text { Cannot download or exxtract to SD card? Please } \\
\text { check Settings }\end{array}$} \\
\hline \multicolumn{3}{|c|}{$\begin{array}{l}\text { G GUI Mode } \\
\text { minimap2 -x map-ont } \\
\text { /storage/emulated/o/mobile-genomics/data-set-1 }\end{array}$} \\
\hline SELECT FOLDER & COPY PATH & PASTE PATH \\
\hline \multicolumn{3}{|c|}{$\square$ Temporary Prefix for multi-part index(--split-prefix )(i) } \\
\hline \multicolumn{3}{|l|}{$\checkmark$ reference index $(i)$} \\
\hline \multicolumn{3}{|c|}{$\begin{array}{l}\text { /storage/emulated/0/mobile-genomics/ } \\
\text { data-set-1/draft.fa }\end{array}$} \\
\hline \multicolumn{3}{|c|}{$\checkmark$ query sequence $(i$} \\
\hline \multicolumn{3}{|c|}{$\begin{array}{l}\text { /storage/emulated/0/mobile-genomics/ } \\
\text { data-set-1/reads.fasta }\end{array}$} \\
\hline \multicolumn{3}{|l|}{$\checkmark$ output file(-o)(i) } \\
\hline \multicolumn{3}{|c|}{$\begin{array}{l}\text { /storage/emulated/0/mobile-genomics/ } \\
\text { data-set-1/min-align.sam }\end{array}$} \\
\hline \multicolumn{3}{|c|}{$\checkmark$ Output SAM format (Default PAF format)(-a) (i) } \\
\hline$\square$ Generate CIGAR a & ecg tag of $P$ & $F(-0)$ (i) \\
\hline
\end{tabular}

B F5N Help

F5N is a Mobile application for Reference Guided Sequence Alignment using Oxford Nanopore Technology Data

Please read the following terms before you use this application

This app needs storage permission in order to read/write files generated from the pipeline steps

This app needs at least 2 GB free RAM to smoothly function

If you have less memory available, minimizing the app hile running some process, may stop the process and generate incomplete results

Refer $\mathbf{5 5 n}$.log in main_storage/mobile-genomics folder to read the log.

Refer tmp.log in main_storage/mobile-genomics folder in cases where the app fails in the middle of a process

\section{VIEW TUTORIAL}

Tool versions used in this app

- Minimap2 v2.17-r974-dirty, commit d2de282

Samtools v1.10, commit $\underline{\underline{5 c a 6 e e} 2}$

F5C v0.3-beta, commit cea $05 f 7$

Please contribute to our work by testing,debugging developing and submitting issues on our produc

\section{E Demo Mode}

This mode will run all the 5 steps(minimap2 alignment samtools sort, samtools index $f 5 \mathrm{c}$ index, $f 5 \mathrm{c}$

call-methylation and 55 ceventalign) on ecoli data set

The App will automaticolly download and extract the data

set and configure the pipeline

All of the files will be in a folder called mobile-genomics in your main storage

This will run all the 5 pipeline steps on Ecoli dataset(10MB)

You can find all the files downloaded, created and the logcat in the mobile-genomics folder in your main storage If you have already downloaded and extracted the ecoli bile-genomics folder you can skip Download \& Extract

DOWNLOAD \& EXTRACT

\section{RUN PIPELINE}

\section{H Terminal Mode}

/storage/emulated///mobile-genomics/data-set-1

\section{SELECT FOLDER COPY PATH PASTE PATH}

minimap2 -x map-ont /storage/emulated/0/ mobile-genomics/data-set-1/draft.fa /storage/ emulated/0/mobile-genomics/data-set-1/ reads.fasta $-0 /$ storage/emulated/0/

mobile-genomics/data-set-1/min-align.sam -a -t 4 -K 20M

\section{NEXT}

change the output file paths to a location in the interna storage if writing to the SD card failed
USE GUI MODE

USE TERMINAL MODE

LOAD PREVIOUS CONFIG

\section{Settings}

Genera

Default Storage Path

/storage/emulated/0/mobile-genomics/

Reference Genome Storage Path

X.fa and X.idx files should be stored in this directory to

automatically generate pipeline commands in CONNECT TO

storage/emulated/0/mobile-genomics/

Permissions

Download / Extract to SD Card

Choose SD Card from here

Reduce Brightness during Pipeline Execution Brightness will reduce once the pipeline starts. Do

not minimize or turn off the display, process may

About

$5 \mathrm{~N}$ is a Mobile application for Reference Guided Sequence

\section{F Pipeline Step Selection}

$\checkmark$ MINIMAP2_SEQUENCE_ALIGNMENT

$\checkmark$ SAMTOOLS_SORT

$\checkmark$ SAMTOOLS_INDEX

$\square$ F5C_INDEX

$\square$ F5C_CALL_METHYLATION

$\square$ F5C_EVENT_ALIGNMENT

$\square$ F5C_METH_FREQ

\begin{tabular}{|c|}
\hline USE GUI MODE \\
\hline USE TERMINAL MODE \\
\hline LOAD PREVIOUS CONFIG \\
\hline
\end{tabular}

\section{Pipeline Execution}

minimap $2-x$ map-ont /storage/emulated/0/

mobile-genomics/data-set-1/draft.fa /storage/emulated/ $0 /$ moble-genomics/data-set- $1 /$ reads.fasta $-0 /$ storage/ -t 4 -K $20 \mathrm{M}$

$$
\text { RUN THE PIPELINE }
$$
00:01

target sequence(s)
[M::mm_mapopt_update::0.619*18.37] mid_occ $=1$

M..mm_mapopt_update...6.619.3.37 mid_occ $=11$ M::mm_idx_stat] kmer size: 15; skip: 10; is_hpc: 0 ; \#seq: 3 (M::.mm_idX_stat::0.653*17.47] distinct minimizers: 83347 (98.00\% are singletons); average occurrences: 1.038 acing
average spacing: 5.355 ; total length: 4620825 [M::worker_pipeline::1.106*11.91] mapped 112 sequences [M:: :init_minimap2] Version: 2.17-r974-dirty [M:init_minimap2] Version: 2.17-r974-dirty [M::init_minimap2] CMD: minimap2 2-x map-ont -0/storage/ emulated/0/mobile-genomics/data-set-1/min-align.sam data-set-1/draft.fa /storage/emulated/0/mobile-genomics/ data-set-1/reads fasta

[M::init_minimap2] Real time: $1.126 \mathrm{sec}$; CPU: $13.220 \mathrm{sec}$; Peak RSS: 0.183 GB

By default, output files are written to the mobile-genomics folder in your main storage. If you have updated the output paths please check the respective folders

WRITE LOG TO FILE

minimap 2 -x map-ont took 01s status $=$ Success

Fig. S3. $\quad F 5 N$ screenshots (A) Homepage. (B) Help page. (C) Set F5N Settings. (D) Download and extract files. (E) Run an example pipeline. (F) Select pipeline steps. (G) Configure pipeline steps using GUI. (H) Configure pipeline steps using terminal environment. (I) Pipeline execution 


\section{DETAILED F5N EXAMPLES AND RESULTS}

In this section, we present a detailed overview of the tests run using $F 5 N$. GRCh38 human genome reference was used as the reference (3.1 GB). For reads alignment, the index constructed for the whole reference by Minimap2 is too large to fit in RAM ( $\sim 8 \mathrm{~GB})$. It was partitioned into 8 partitions using the script [13]. The resulting partitioned index is $\sim 8.2$ GB in size. Since now only a single partition is loaded to RAM at once, the memory of a mobile device is sufficient for Minimap2 alignment. User has to store both the reference genome and its partitioned version either on the internal storage or on the external storage (SD card). Please refer Supplementary Section VIII on how to choose the SD card format. Two datasets, flowcell id-FAB42804 and flowcell id-FAF05869 were downloaded from the publicly available NA12878 nanopore sequenced data repository [10].

The first dataset (FAB42804) had 16688 reads (91.15 Mbases) and the second dataset had 451,359 reads (3.89 Gbases). The first dataset was used as is to execute a complete methylation calling pipeline. To mimic the batch processing behaviour of the basecaller, the second dataset (FAF05869) was divided into batches of 4000 reads ( 34.49 Mbases per batch on average). For this, the original raw signal files (single-fast5 files) of FAF05869 were downloaded and converted to multi-fast5 using ONT's single_to_multifast5 tool (version 1.4.8) with default options. Then, these multi-fast5 files were re-basecalled using ONT's basecaller guppy (version 3.3.0) under the dna_r9.4.1_450bps_hac model.

The devices listed in the table S1 were used in the experiments. All the devices were octa-core arm64-v8a instruction set devices. Methylation calling pipeline illustrated in the Supplementary Section I was executed on the first dataset and on batches from the second dataset ( 2 batches per device). The datasets along with the reference and partitioned index were copied to mobile devices through a USB connection. Each test was repeated 3 times on a device and the tables S2-S5 show the average values. The results are discussed in the paper.

Below, are the commands ran on a device to execute the complete methylation pipeline. These commands were generated through F5N's GUI mode, except for the first command.

1) Partitioning the reference genome (on a computer)

divide_and_index.sh [reference.fa file] [num_parts [8]] [output.idx file] [min-

imap2_exe] [minimap2_profile [map-ont] ]

2) Aligning reads to the partitioned human genome using Minimap2

minimap2 -x map-ont --split-prefix [temporary file path] [partitioned reference]

[reads.fastqlfasta] -o [output .sam file] -a -t [no. of threads [4]] -K [Number of bases

loaded into memory to process in a mini-batch [5M]]

3) Sort aligned reads using Samtools

samtool sort [minimap2 output] -o [output .bam file]

4) Index sorted reads using Samtools

samtool index [samtools sort output]

5) Index fast5 files

f5c index --directory [fast 5 _folder] [reads.fastqlfasta]

6) DNA methylation calling using F5C

f5c call-methylation -r [reads.fastqlfasta] -b [path to samtools index output] -g [ref.fa] -o [output meth.tsv] -B [max number of bases loaded at once [2.0M]] -K [max number of reads loaded at once [256]]

7) Aligning nanopore events to reference k-mers using F5C (optional, not a step in methylation calling)

f5c eventalign -r [reads.fastqlfasta] -b [path to samtools index output] -g [ref.fa] -o [output meth.tsv] -B [max number of bases loaded at once [2.0M]] -K [max number of reads loaded at once [256]] --summary [output events.summary.txt]

TABLE S1. LIST OF MOBILES USED IN THE EXPERIMENTS

\begin{tabular}{|c|c|c|c|c|}
\hline Device & Manufacturer & Model & RAM(GB) & Internal Storage(GB) \\
\hline A & Huawei & Nova 5T & 8 & 128 \\
B & Nokia & 6.1 Plus & 4 & 64 \\
C & Samsung & A70 & 8 & 120 \\
D & Xiaomi & Mi 9T & 6 & 120 \\
\hline
\end{tabular}

TABLE S2. F5N PERFORMANCE FOR THE COMPLETE NA12878-FAB42804 DATASET (91 MBASES)

\begin{tabular}{|c|c|c|c|c|c|c|}
\hline Device & Minimap2 (min) & Samtool sort (min) & Samtool index (min) & F5C index (min) & F5C call methylation (min) & Total time (min) \\
\hline A & 18.52 & 0.20 & 0.02 & 0.37 & 2.37 & 2.82 \\
B & 14.48 & 0.38 & 0.02 & 0.70 & 4.45 & 20.40 \\
C & 16.67 & 0.36 & 0.03 & 0.65 & 3.58 & 22.68 \\
D & 16.27 & 0.28 & 0.02 & 0.53 & 20.18 \\
\hline
\end{tabular}

TABLE S3. PEAK RAM OF INDIVIDUAL STEPS WHEN PROCESSING NA12878-FAB42804 DATASET

\begin{tabular}{|c|c|c|c|c|c|}
\hline Device & Minimap2 (GB) & Samtool sort (GB) & Samtool index (GB) & F5C index (GB) & F5C call methylation (GB) \\
\hline A & 2.20 & 0.32 & 0.19 & 0.15 & 3.95 \\
B & 2.22 & 0.26 & 0.09 & 0.10 & 2.78 \\
C & 2.27 & 0.30 & 0.14 & 0.15 & 3.58 \\
D & 2.22 & 0.34 & 0.18 & 0.19 & 3.28 \\
\hline
\end{tabular}

\section{ADJUSTING MEMORY GOVERNING PARAMETERS OF THE TOOLS}

The following steps can reduce peak RAM usage, to run $F 5 N$ on a broad spectrum of mobile devices. For more details visit Minimap2 man page[14], Samtools man page[15] and F5C man page[16]. 
bioRxiv preprint doi: https://doi.org/10.1101/2020.03.22.002030; this version posted June 9, 2020. The copyright holder for this preprint (which was not certified by peer review) is the author/funder, who has granted bioRxiv a license to display the preprint in perpetuity. It is made available under aCC-BY-NC 4.0 International license.

TABLE S4. F5N PERFORMANCE FOR A BATCH OF 4K READS (34 MBASES) FROM NA12878-FAF05869 DATASET

\begin{tabular}{|c|c|c|c|c|c|c|}
\hline Device & Minimap2 (min) & Samtool sort $(\mathrm{min})$ & Samtool index $(\min )$ & F5C index (min) & F5C call methylation (min) & Total time (min) \\
\hline A & 11.83 & 0.07 & 0.02 & 0.13 & 1.13 & 13.18 \\
B & 13.20 & 0.13 & 0.02 & 0.25 & 2.98 & 16.58 \\
C & 11.02 & 0.13 & 0.02 & 0.25 & 14.25 \\
D & 10.23 & 0.10 & 0.02 & 0.17 & 1.82 & 12.34 \\
\hline
\end{tabular}

TABLE S5. PEAK RAM OF INDIVIDUAL STEPS WHEN PROCESSING A BATCH OF 4K READS FROM NA12878-FAF05869 DATASET

\begin{tabular}{|c|c|c|c|c|c|}
\hline Device & Minimap2 (GB) & Samtool sort (GB) & Samtool index (GB) & F5C index (GB) & F5C call methylation (GB) \\
\hline A & 2.48 & 0.16 & 0.09 & 0.10 & 0.99 \\
B & 3.16 & 0.22 & 0.14 & 0.16 & 1.18 \\
C & 3.16 & 0.22 & 0.14 & 0.16 & 1.18 \\
D & 3.41 & 0.23 & 0.16 & 0.17 & 1.19 \\
\hline
\end{tabular}

1) Increase the number of partitions the genome reference is split into, to reduce peak RAM usage in Minimap2 alignment

2) In Minimap2 alignment reduce the parameter value, number of bases loaded into memory to process in a mini-batch [-K]

3) In Samtools sort reduce the parameter value, the maximum required memory per thread [- $m$ ]

4) In F5C call methylation reduce the parameter value, batch size (max number of reads loaded at once) [-K]

5) In F5C call methylation reduce the parameter value, max number of bases loaded at once [-B]

6) In F5C call methylation skip ultra long reads by setting the option [-skip-ultra FILE]

7) In F5C call methylation reduce the threshold value to skip ultra long reads [-ultra-thresh INT]

\section{CAClulating BASE-CALling RATE}

Given the total number of reads in a dataset $N$, the number of reads in a base-called batch $B$ and the duration of sequencing run $T$, we can calculate the average time taken by the basecaller to produce a batch (base calling rate) $R$ using the following equation,

$$
R=\frac{T B}{N}
$$

In our case $T=48$ hours, $B=4000$ and $N=451,359$ which results in $R$ to be 25.52 min. 


\section{RECONSTRUCTING SEQUENCE ANALYSIS TOOLS FOR ARM/ANDROID}

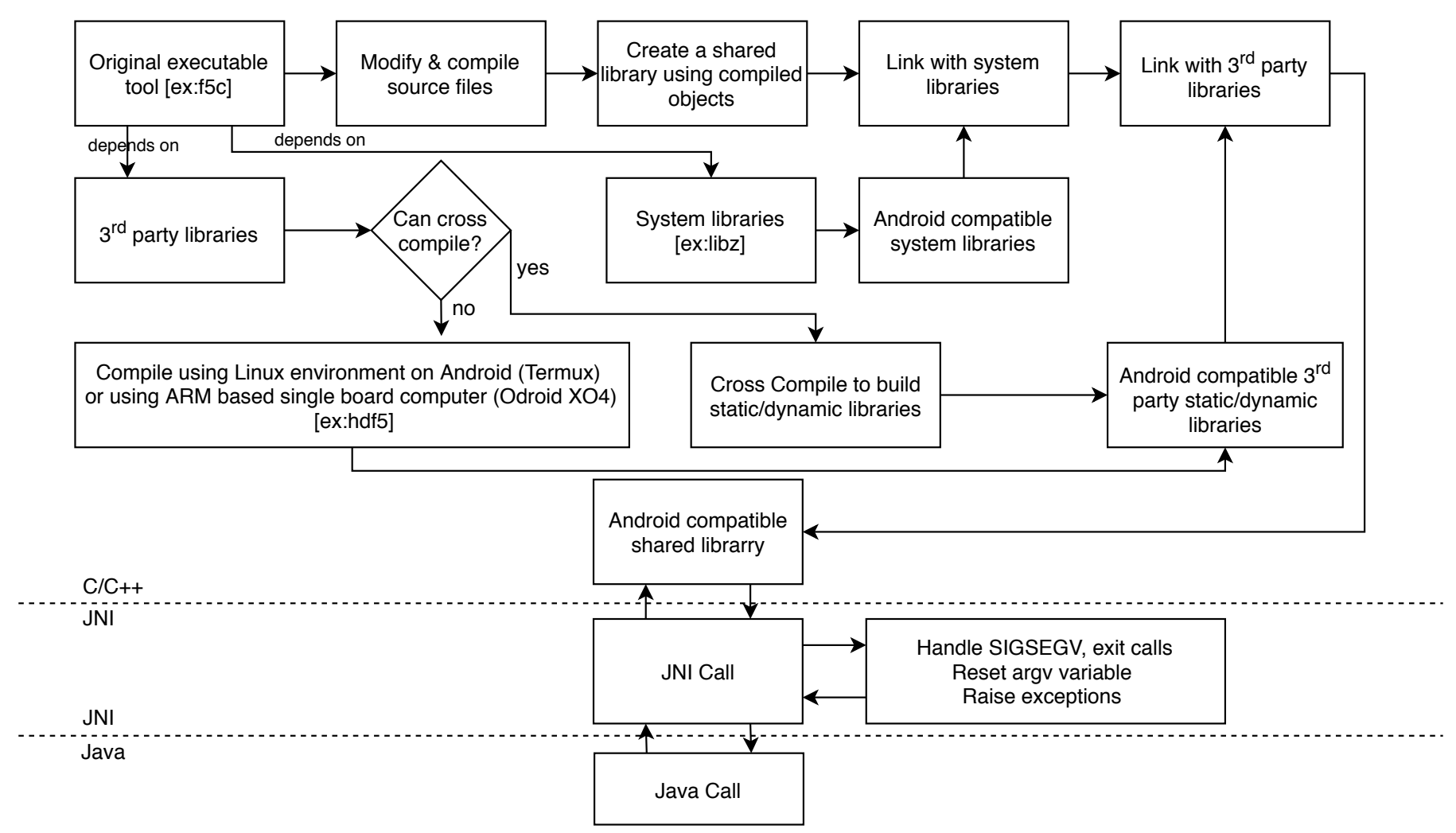

Fig. S4. Cross-compiling work flow and JNI interface design involved in including a new sequence analyis tool to $F 5 N$.

\section{A. Introduction and prerequisites}

This section provides fundamental guidelines on how to compile an analysis tool written in $\mathrm{C} / \mathrm{C}++$ for Android. Except for a few essential amendments (discussed below), our approach does not alter the original $\mathrm{C} / \mathrm{C}++$ code, which permits straightforward integration of new $\mathrm{C} / \mathrm{C}++$ analysis tools into $F 5 N$.

Having some prior experience with Android SDK (Software Development Kit), Android Studio[17], NDK (Native Development Kit) [18], Java Native Interface (JNI)[19], ADB (Android Debug Bridge)[20], CMake build manager[21] and Ninja build system[22] certainly helps someone who is interested in rebuilding $F 5 N$ or extending it with new tools.

\section{B. Native compiling an analysis tool}

In our case, the fact that all the necessary tools (Minimap2, Samtools and F5C) were written in $\mathrm{C} / \mathrm{C}++$ let us to follow the same approach to compile them for Android. The approach can be summarized as shown in Fig. S4. Before starting the compilation we tested the tools using Termux (a terminal emulator with Linux environment for Android)[23]. In Termux, the tools' git repositories were cloned and built as instructed on the respective installation guides. This method can be known as native compiling. If a tool gets natively compiled in Termux, we can reasonably expect the tool to get cross-compiled for Android. There are two major Instruction Set Architectures (ISA) for ARM mobile devices called armeabi-v7a and arm64-v8a. Termux was installed on multiple devices with different architectures to make sure that the tools can be natively compiled in both the architectures. It is noteworthy that in Termux, the executable versions (.exe format) of the tools were built in contrast to their dynamic library versions (.so format). To build an Android application, the dynamic version of an original tool is required[24]. All the Android applications are a subset of Java programs and the gateway to the native $(\mathrm{C} / \mathrm{C}++)$ code is obtained by loading the dynamic version of the native tool. To obtain a dynamic version of the tool (.so format), we can change the build configuration and natively compile in Termux. However, this method is not encouraged as it can impose device based restrictions on .so files which can result in intensive and costly debugging. The recommended method to obtain the dynamic version of a tool is to cross-compile using the Android Tool-chain[25]. This eliminates the burden associated with native compiling and simplifies the process of updating the tools to their latest versions. This in return automates continuous integration and delivery. In Suppliementary Sections VI-E and VII we provide further details on how to cross-compile.

\section{Determining third party libraries used by an analysis tool}

It is necessary to determine the third party libraries used by the analysis tools (Minimap2, Samtools and F5C). A tool to function correctly, associated third party libraries should also be linked statically or dynamically with the tool, i.e., third party libraries should also be cross-compiled. Samtools depends on htslib [26] library. F5C depends on both htslib and HDF5 [27] libraries. Hdf5 library is used to handle fast5 files and there exists no straightforward method to cross compile hdf5. Hence we used native compiled instances of HDF5 library (the instances that were built using Termux). We maintained two instances of 
HDF5, one for each Architecture (armeabi-v7a and arm64-v8a). These third party libraries were statically linked to respective shared libraries. If a third party library is being used by two dependent tools, make sure to link a dynamic version of the library. For an example in our case, htslib was used by Samtools and F5C. Trying to statically link htslib separately to each tool, caused the software to crash on some devices. This was resolved by linking htslib dynamically.

\section{Designing JNI interface}

JNI acts as the bridge between native $(\mathrm{C} / \mathrm{C}++)$ methods and Java function calls. In JNI interface, each tool's int main(int $\left.\operatorname{argc}, \operatorname{char}^{*} \operatorname{argv}[]\right)$ function was called. The function name, int main(int $\left.\operatorname{argc}, \operatorname{char} * \operatorname{argv}[]\right)$ was renamed as int init_X(int argc, char* argv []) where X was Minimap2,Samtools or F5C. This function renaming is necessary as the tools are not stand-alone executable applications but dynamic libraries. Moreover, a header file was introduced for each tool that contained the function signature int init_X(int $\left.\operatorname{argc}, \operatorname{char}^{*} \operatorname{argv}[]\right)$. JNI interface was extended to facilitate the following,

1) Handle exit signals returned by native code.

2) Handle SIGSEGV signals returned by native code.

3) Raise exceptions on behalf of the native functions.

4) Reset $\operatorname{argv}$ variable before calling another native function [int init_X(int argc, char* $\operatorname{argv}[])$ ].

5) A tunnel between the native code and the Java program to communicate standard error messages.

6) If original code does not define an output file path argument, redirect standard ouput to a file.

It is important to handle different types of signals and errors thrown by the native code to prevent JVM from crashing. For example the original code most probably will exit with an error if something goes wrong. This kills the JVM if not handled. We want to keep the JVM running throughout a $F 5 N$ session. In order to do that, the exit call should be caught and handled in JNI [28]. In a similar manner, SIGSEGV signals should be handled safely [29]. Once an exit call or SIGSEGV signal is handled this should be informed to Java program so that the user can investigate the problem. To do this, exceptions are thrown from JNI to Java [30].

The original $\mathrm{C} / \mathrm{C}++$ tools take input as command line arguments. When a pipeline with more than one step is executed, the native code attempts to read the same argument vector multiple times. If the argument vector is not reset after the completion of the first step, arguments do not get parsed in the second step as desired. This resetting part is not implemented in most of the original libraries. In JNI this should be implemented to avoid failures associated with arguments parsing [31].

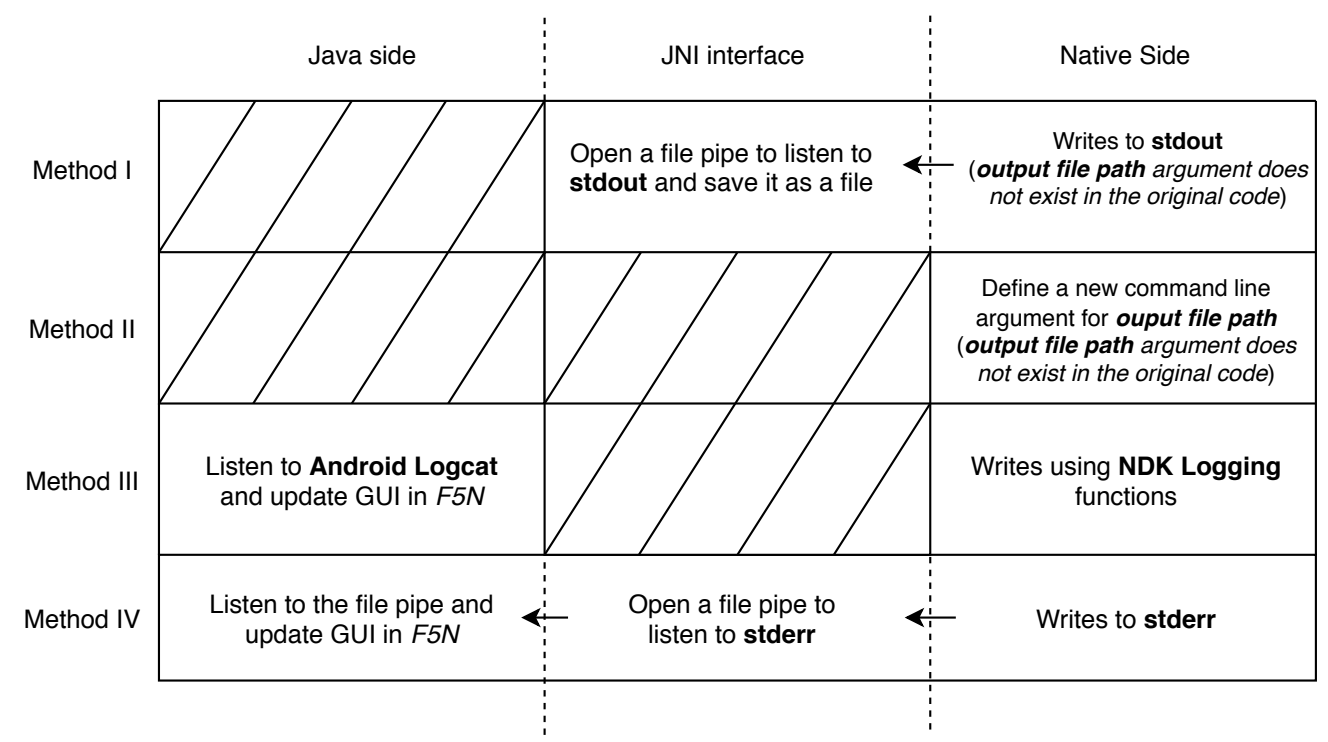

Fig. S5. Method I - Using a file pipe to listen and save standard output. Method II - Define a new command line argument for output file path. Method III Using NDK Logging functions to print standard error to Logcat. Method IV - Using a file pipe to listen standard error.

Typically a tool writes the results to the standard ouput and meta-information to the standard error. On Android, we want the results to be written to a file and meta-information to be displayed on a GUI in real-time. To write results to a file, most of the tools provide a command line argument called file output path. Otherwise, in terminal environments like in Linux, we can redirect the standard output to a file (using the output redirection operator ' $>$ '). On Android, this is not possible. To overcome this issue two methods can be adopted. The first is to open up a file pipe in JNI to listen to the standard ouput, where the results will get written to this file (Fig. S5 Method I). The second is to define the output file path as an argument in the original code (Fig. S5 Method II). However, all the libraries in F5N had output file path as an argument. Now we present two methods to catch the standard error, which should be displayed to the user in real-time. The first method is to replace all the fprintf(stderr,...) functions with functions defined in NDK logging [32]. Then the standard error will get written to Android Logcat [33]. From Android Logcat, it is again piped to be displayed (Fig. S5 Method III). In practice, this method does not guarantee to display the 
complete set of messages written to the standard error during an execution. The more robust method is to open a file descriptor to listen to the standard error. Then this file should be read in Java side and the GUI should be updated (Fig. S5 Method IV). This involves no amendments in the original libraries but a declaration of file descriptors in JNI code.

\section{E. Cross-compiling an analysis tool}

To facilitate the modifications discussed above, the original repositories of the tools were forked and changed. Different tools use different build configurations. The build scripts for each tool were re-written using CMake. Suppose an original tool is built with GNU Make using a Makefile. In that case, one has to extract the source files, header files, compiler flags, linked libraries etc to create a CMakeLists.txt file. Refer how a CMakeLists.txt was written for htslib [34]. CMake along with Ninja is the recommended native build setup for Android. Compiling with CMake allows ADB to go deep into the native code when debugging. This was really helpful to figure out the static/dynamic version issue related to htslib library. It was straightforward to link the necessary third party libraries(HDF5 and htslib) and system libraries (libz, liblog, libm etc) with original libraries using CMake. To follow the full set of modifications please refer Minimap2 [35] Samtools [36] and F5C [37]. One can build libraries without using CMake but by using already available Standalone Toolchains[38]. However, this eliminates the possibility to debug using ADB.

\section{F. Dynamic object construction in Java side}

The part of $F 5 N$ written in Java is dynamic and adaptive. For example the arguments set for each tool is stored in JSON format and objects are created by converting JSON objects to Java objects. In this way, $F 5 \mathrm{~N}$ and arguments are decoupled making it easy to alter the format of the arguments if needed. The widgets linked with arguments are drawn programmatically instead of manually drawing them on the layout. This makes it easy to extend $F 5 N$ with new analysis tools (refer Supplementary Section VII).

\section{INTEGRATING A NEW ANALYSIS TOOL TO F5N}

The following steps summarize the work flow to add a new DNA analysis tool written in C/C++ to F5N. Please refer Fig. S6 for $F 5 N$ directory structure. $F 5 N$ repository is available at https://github.com/SanojPunchihewa/f5n

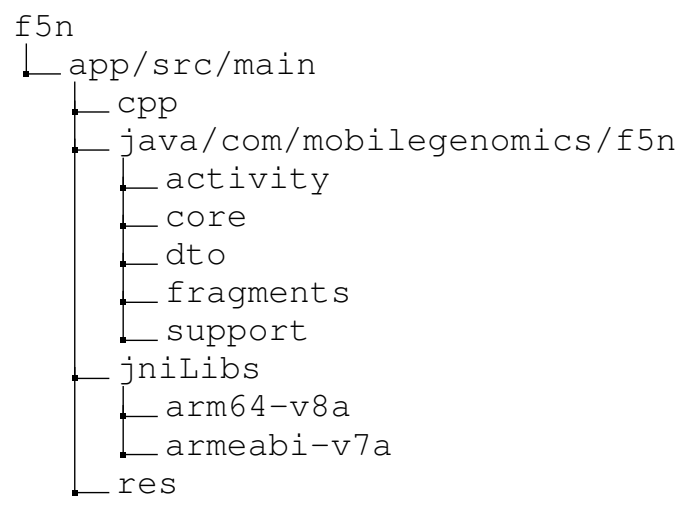

Fig. S6. F5N project directory structure, only the important folders are shown. The directory cpp contains CMakeLists.txt and interface_X.h header files. The .so files are stored according their ISA inside jniLibs directory. The Java Class PipelineStep is inside directory core.

1) Identify third party libraries that the new tool depends on.

e.g. Samtools depends on htslib.

2) Create dynamic versions of third party libraries if they are used by other analysis tools in $F 5 N$.

3) Create static versions of third party libraries if they are not used by other analysis tools in $F 5 N$.

4) Cross compile the new tool and link it with static third party libraries to create a dynamic library.

5) Place all the dynamic libraries in jniLibs/[ANDROID_ABI] directory.

6) Repeat the above steps for different ANDROID_ABIs. (armeabi-v7a and arm64-v8a)

7) Change CMakeLists.txt file in cpp directory to include dynamic third party dependencies.

- add_library (libnewdependency SHARED IMPORTED)

- set_target_properties(libnewdependency PROPERTIES IMPORTED_LOCATION CMAKE SOURCE DIR/../jniLibs/\$ANDROID ABI/libnewdependency. so)

8) Change CMakeL Lists.txt to include the new analysis library.

- add_library (libnew SHARED IMPORTED)

- set_target_properties(libnew PROPERTIES IMPORTED_LOCATION CMAKE SOURCE DIR/../jniLibs/\$ANDROID ABI/libnew. so)

9) Change CMake $\bar{L}$ ists.txt file to link the new analysis library.

- target link libraries (native-lib libminimap libsamtool libf5c

libhts libnew libnewdependencyl libnewdependency2 ...\$log-lib)

10) Copy interface_X.h file with the function signature init_X(int $\operatorname{argc}$, char* $\operatorname{argv}[]$ ) to $\operatorname{cpp}$ directory ( $\mathrm{X}$ is the name of the new library).

11) Create enum entries in PipelineStep Class located in core directory for the sub-tools (commands) in the new analysis tool.

- NEW_TOOL_NAME (COMMAND_ID, "tool_name sub-tool_name (command)"); 
ex: F5C INDEX (3, "f5c index");

12) For each sub-tool (command) create a JSON file containing the list of arguments and their default values[39].

13) Link JSON files to the configureArguments method in GUIConfiguration Class located inside java/com/mobilegenomics/f5n directory, i.e. add new switch cases to match NEW_SUB_TOOLs and assign JSON files appropriately.

14) Import interface_X.h header file to native-lib.cpp source file located inside cpp directory.

- include "\#interface_X.h"

15) Follow a similar approach to other tools to call init_X(int argc, char*argv[]) function in native-lib.cpp source file.

Moreover, the following list comprises of common mistakes that could happen when extending or rebuilding $F 5 N$. Please refer Supplementary Section VI for details.

1) Overlooking compiler flags when creating a CMake build configuration.

e.g. not including the compiler flag -D_FILE_OFFSET_BITS=64 in Minimap2 CMake build will result in file read failures.

2) Statically linking third party libraries that are used by more than one tool.

e.g. Linking the static version of htslib library caused Samtools and F5C to fail.

3) Not Handling native exceptions in JNI interface

4) Not Handling native SIGSEGV signals and exit signals in JNI interface

5) Not Resetting command line argument vector before calling a new native function

\section{ADVANCED DETAILS}

$F 5 N$ is not built as an Android background process. That is because when processing a dataset, $F 5 N$ may consume memory more than the recommended amount for a background process. Android kills such over memory consuming background processes. Hence, $F 5 N$ is built as a regular Android application. However, this introduced a caveat. That is when running a pipeline, the device display should be kept on. That is because Android interrupts running applications once the display goes off. Hence, $F 5 N$ has to keep the display on and this increases power consumption. Our workaround is to reduce the display brightness to its minimal value, once a pipeline execution starts. With this method, device B's battery (3060 mAh) drains approximately by $214.2 \mathrm{mAh}$ for a complete methylation calling pipeline on a batch data-set ( 34.49 Mbases on average).

Android tends to kill a process or destroy an activity if something goes wrong. In $F 5 N$ usually it the case when the native code tries to over consume memory. Since this kill signal comes from the Android Kernel, it cannot be handled. The simple solution is to be aware of the device memory and set memory governing parameters accordingly (see Supplementary Section IV). Saving the state of the application, i.e, saving previously executed command and loading it later, saves time for re-configuring the pipeline (Fig. S3F). In the next attempt, the user is advised to tweak memory parameters as it is the solution most of the time.

Already executing native code on Android cannot be stopped arbitrarily. The ramification of this is that the user cannot suspend or stop an executing pipeline. On the other hand, JVM being a multi-threaded process and JNI calls not being POSIX asyncsignal-safe, it is not possible to run the native code in a cloned process. As a consequence peak RAM usage will record the highest RAM usage for the whole application session rather than for the latest pipeline execution. To circumvent both problems the "safest" solution is to restart F5N. An advanced method to suspend or stop an executing command is by adding interrupt listeners to the original code. That is while the original code is being executed, it periodically checks its environment for an interrupt signal, e.g, state of a flag value in a file. The flag value can be changed on-the-fly to stop or suspend the execution. Please note that in F5N, when a pipeline is running the user can still go to the previous activity. In such attempts, a warning message is displayed saying that the pipeline will stop. This does not guarantee the complete termination of the native process but the termination of the running Java thread. Hence, the user is advised to restart the application to safely terminate a pipeline.

A mobile phone has two storage types - internal storage and external storage (SD card storage). FAT32 is a popular file system format used in SD cards. However, FAT32 does not support files with more than the size of $\sim 4 G B$. Usually, the partitioned genome reference file exceeds the size 4GB. Therefore, the user is advised to use file system formats like ext3, ext4, exFAT32 etc as the SD card file system format. Out of these formats, exFAT32 is recommended. It is noteworthy that Google has introduced (from Android 8.0) a virtual file system wrapper called SDCardFS to regulate SD card access by Android applications. However, still, the SD card should have one of the compatible file system formats to work with larger files.

Downloading and extracting a dataset to the SD card can be done after setting SD card permission (refer Fig S3C). On Android, writing to the SD card storage via native code $(\mathrm{C} / \mathrm{C}++)$ can only be done using Storage Access Framework (SAF)[40]. To implement SAF, most of the original code has to be changed. One workaround is to use the Method I as illustrated in Fig. S5. In JNI interface, we can use SAF to write the standard output to the SD card. However, there are sub-tools that directly write to files, e.g., F5C index writes files automatically to the dataset directory. In such scenarios, the original tool should be reconfigured to facilitate SAF. The current version of $F 5 N$ does not support this feature. Therefore the user cannot perform writes to the SD card but can read from it.

\section{SUPPLEMENTARY REFERENCES}

[11] Heng Li. "Minimap and miniasm: fast mapping and de novo assembly for noisy long sequences". In: Bioinformatics 32.14 (2016), pp. 2103-2110.

[12] Jared Simpson. Nanopolish. https:https://github.com/jts/nanopolish.

[13] Hasindu Gamaarachchi. Index construction with chromosome size balancing. https://github.com/hasindu2008/minimap2arm/tree/master/misc/idxtools.

[14] Heng Li. Manual Reference Pages - Minimap2 (1). https://lh3.github.io/minimap2/minimap2.html.

[15] Samtools. Manual page from samtools-1.10. http://www.htslib.org/doc/samtools.html.

[16] Hasindu Gamaarachchi. Manual Reference Pages - F5C. https://hasindu2008.github.io/f5c/docs/overview.

[17] Android. Android Studio and SDK. https://developer.android.com/studio.

[18] Android. Android NDK. https://developer.android.com/ndk. 
bioRxiv preprint doi: https://doi org/10.1101/2020.03.22 002030; this version posted June 9,2020 . The copyright holder for this preprint (which was not certified by peer review) is the author/funder, who has granted bioRxiv a license to display the preprint in perpetuity. It is made available under aCC-BY-NC 4.0 International license.

[19] Android. JNI. https://developer.android.com/training/articles/perf-jni.

[20] Android. Android Debug Bridge. https://developer.android.com/studio/command-line/adb.

[21] Kitware. CMake build manager. https://cmake.org/.

[22] Ninja. Ninja build system. https://ninja-build.org/.

[23] Fredrik Fornwall. Termux Linux environment emulator. https://play.google.com/store/apps/details?id=com.termux\&hl=en.

[24] Ian F Darwin. Android Cookbook: Problems and Solutions for Android Developers. " O'Reilly Media, Inc.", 2017, "661$666 "$.

[25] Android. Android Cmake cross-compilation. https://developer.android.com/ndk/guides/cmake.

[26] samtools. htslib. https://github.com/samtools/htslib.

[27] Mike Folk, Albert Cheng, and Kim Yates. "HDF5: A file format and I/O library for high performance computing applications". In: Proceedings of supercomputing. Vol. 99. 1999, pp. 5-33.

[28] JNI handle exit calls. http://jnicookbook.owsiak.org/recipe-no-016/.

[29] JNI handle SIGSEGV calls. http://jnicookbook.owsiak.org/recipe-no-015/.

[30] JNI throw exceptions. http://jnicookbook.owsiak.org/recipe-no-019/.

[31] Sanoj Punchihewa. F5N JNI interface. https://github.com/SanojPunchihewa/f5n/blob/master/app/src/main/cpp/nativelib.cpp.

[32] Android. Logging Android NDK. https://developer.android.com/ndk/reference/group/logging.

[33] Android. Logcat command-line tool. https://developer.android.com/studio/command-line/logcat.

[34] HTSLIB. CMake build for HTSLIB. https : / / github . com / hiruna72 / htslib / blob / 76f9eaa29a23573a70e37ca6ed842719e03cde55/INSTALL\#L101.

[35] Minimap2. CMake build for Minimap2. https://github.com/SanojPunchihewa/minimap2-arm/tree/build-cmake\#cmakebuild.

[36] Samtools. CMake build for Samtools. https : / / github . com / hiruna72 / samtools / blob / 947c5b66cf91abc9b3e58b61642994dd8f4ae7e4/INSTALL\#L103.

[37] F5C. CMake build for F5C. https://github.com/hiruna $72 / f 5 \mathrm{c} /$ tree/cmake_build\#building.

[38] Android. Standalone Toolchains (Obsolete). https://developer.android.com/ndk/guides/standalone_toolchain.

[39] F5N. F5N arguments JSON format. https://github.com/SanojPunchihewa/f5n/blob/master/app/src/main/res/raw/minimap2. json.

[40] Android. Open files using storage access framework. https://developer. android.com/guide/topics/providers/documentprovider.html. 